\title{
Multi-modality Sensor Data Classification with Selective Attention
}

\author{
Xiang Zhang ${ }^{\dagger}$, Lina Yao $^{\dagger}$, Chaoran Huang ${ }^{\dagger}$, \\ Sen Wang ${ }^{\S}$, Mingkui Tan`, Guodong Long ${ }^{\ddagger}$ and Can Wang ${ }^{\S}$ \\ $\dagger$ School of Computer Science and Engineering, University of New South Wales \\ $\S$ School of Information and Communication Technology, Griffith University \\ School of Software Engineering, South China University of Technology \\ $\ddagger$ Center for Quantum Computation and Intelligent Systems, University of Technology Sydney \\ xiang.zhang3@student.unsw.edu.au, \{lina.yao, chaoran.huang $\} @$ unsw.edu.au, \\ \{sen.wang, can.wang\}@griffith.edu.au, mingkuitan@scut.edu.cn, guodong.long@uts.edu.au
}

\begin{abstract}
Multimodal wearable sensor data classification plays an important role in ubiquitous computing and has a wide range of applications in scenarios from healthcare to entertainment. However, most existing work in this field employs domain-specific approaches and is thus ineffective in complex situations where multi-modality sensor data are collected. Moreover, the wearable sensor data are less informative than the conventional data such as texts or images. In this paper, to improve the adaptability of such classification methods across different application domains, we turn this classification task into a game and apply a deep reinforcement learning scheme to deal with complex situations dynamically. Additionally, we introduce a selective attention mechanism into the reinforcement learning scheme to focus on the crucial dimensions of the data. This mechanism helps to capture extra information from the signal and thus it is able to significantly improve the discriminative power of the classifier. We carry out several experiments on three wearable sensor datasets and demonstrate the competitive performance of the proposed approach compared to several state-of-the-art baselines.
\end{abstract}

\section{Introduction}

Nowadays, diverse categories of sensors can be found in various wearable devices. Such devices are now being widely applied in multiple fields, such as Internet of Things [Kamburugamuve et al., 2015; Zhang et al., ]. As a result, massive multimodal sensor data are being produced continuously. The question that how we can deal with these data efficiently and effectively has become a major concern.

Compared to conventional sensor data such as images and videos, these data are naturally formed as a 1-D signal, with each element representing one sensor channel accordingly. There are several challenges for such sensor data classification. First, most existing classification methods use domain- specific knowledge and thus may become ineffective or even fail in complex situations where multimodal sensor data are being collected [Bigdely-Shamlo et al., 2015]. For example, one approach that works well on IMU (Inertial Measurement Unit) signals may not be able to deal with EEG (Electroencephalography) brain signals. Therefore, an effective and universal sensor data classification method is highly desirable for complex situations. This framework is expected to have both efficiency and robustness over various sensor signals.

Second, the wearable sensor data carries far less information than texts and images. For example, a sample signal gathered by a 64-channel EEG equipment only contains 64 numerical elements. Hence, a more effective classifier is required to extract discriminative information from such limited raw data. However, maximizing the utilization of the given scarce data demands cautious preprocessing and a rich fund of domain knowledge.

Inspired by attention mechanism [Cavanagh and others, 1992], we propose to concentrate on a focal zone of the signal to automatically learn the informative attention patterns for different sensor combinations. Here, the focal zone is a selection block of the signal with a certain length, sliding over the feature dimensions. Note that reinforcement learning has been shown to be capable of learning humancontrol level policy on a variety of tasks [Mnih et al., 2015]. Then we exploit the reinforcement learning to discover the focal zone. Moreover, considering that the signals in different categories may have different inter-dimension dependency [Markham and Townshend, 1981], we propose to use the LSTM (Long Short-Term Memory [Gers et al., 1999; Zhang et al., 2018]) to exploit the latent correlation between signal dimensions. We propose a weighted average spatial LSTM (WAS-LSTM) classifier by exploring the dependency in sensor data.

The main contributions of this paper are as follows:

- We propose a selective attention mechanism for sensor data classification using the spatial information only. The proposed method is insensitive to sensor types since it is capable of handling multimodal sensor data.

- We apply deep reinforcement learning to automatically 


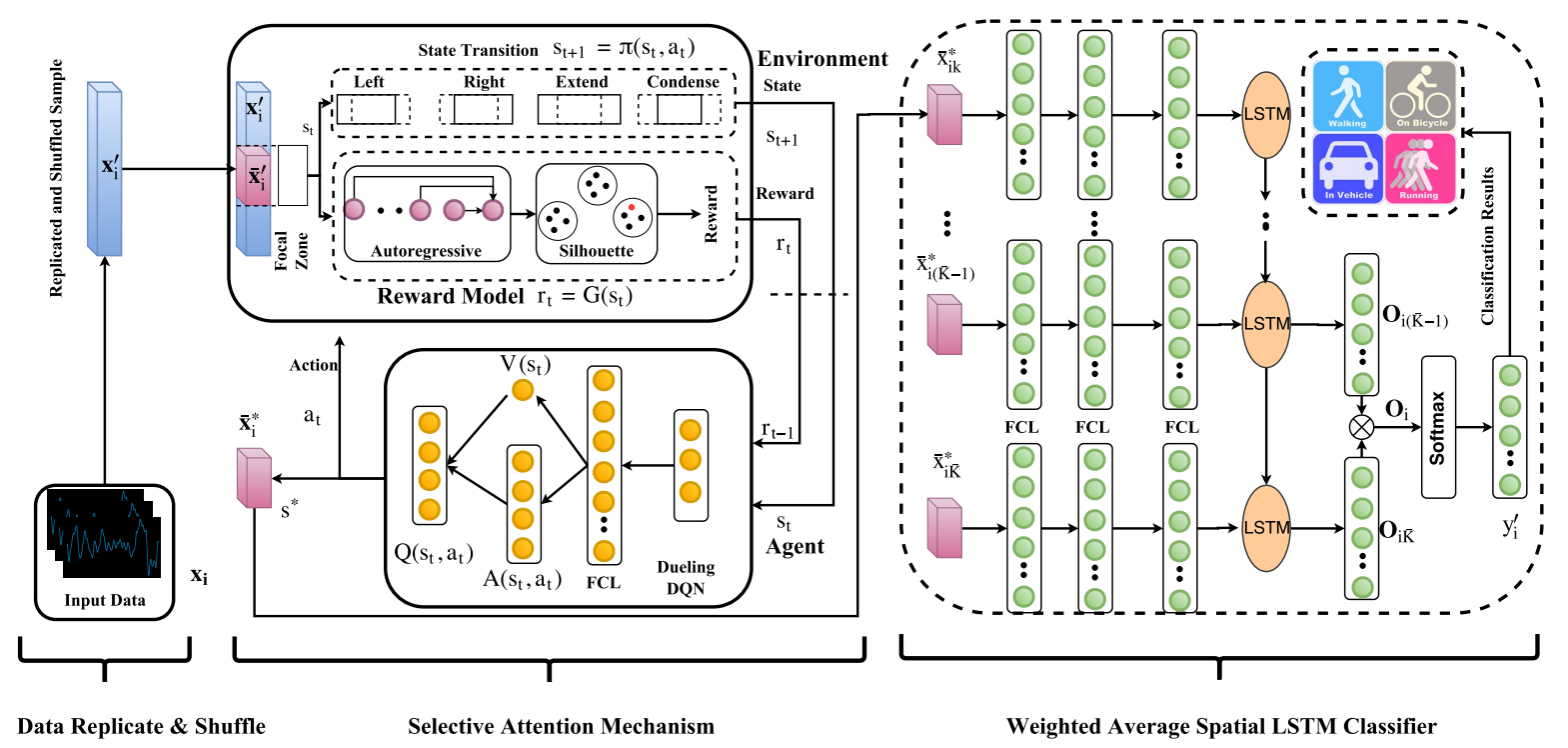

Figure 1: Flowchart of the proposed approach. The focal zone $\overline{\mathbf{x}}_{i}$ is a selected fragment from $\mathbf{x}_{i}^{\prime}$ to feed in the state transition and the reward model. In each step $t$, one action is selected by the state transition to update $s_{t}$ based on the agent's feedback. The reward model evaluates the quality of the focal zone to the reward $r_{t}$. The dueling DQN is employed to find the optimal focal zone $\overline{\mathbf{x}}_{i}^{*}$ which will be feed into the LSTM based classifier to explore the inter-dimension dependency and predict the sample's label $y_{i}^{\prime}$. FCL denotes Fully Connected Layer. The State Transition contains four actions: left shifting, right shifting, extend, and condense.

select the most distinguishable features, called focal zone, for multimodal sensor data of different sensor types and combinations. We design a novel objective function as the award in reinforcement learning task to optimize the focal zone. The new reward model saves more than $98 \%$ training time of the deep reinforcement learning.

- We propose Weighted Average Spatial LSTM classifier to capture the cross-dimensional dependency in multimodal sensor data.

\section{Proposed Method}

Suppose the input sensor data can be denoted by $\mathbf{X}=$ $\left\{\left(\mathbf{x}_{i}, y_{i}\right), i=1,2, \cdots I\right\}$ where $\left(\mathbf{x}_{i}, y_{i}\right)$ denotes the 1-D sensor signal, called one sample in this paper, and $I$ denotes the number of samples. In each sample, the feature $\mathbf{x}_{i} \in \mathbb{R}^{K}$ contains $K$ elements and the corresponding ground truth $y_{i} \in \mathbb{R}$ is an integer denotes the sample's category. $\mathbf{x}_{i}$ can be described as a vector with $K$ elements, $\mathbf{x}_{i}=\left\{x_{i k}, k=\right.$ $1,2, \cdots, K\}$.

The proposed algorithm is shown in Figure 1. The main focus of the algorithm is to exploit the latent dependency between different signal dimensions. To this end, the proposed approach contains several components: 1) the replicate and shuffle processing; 2) the selective attention learning; 3) the sequential LSTM-based classification. In the following, we will first discuss the motivations of the proposed method and then introduce the aforementioned components in details.

\subsection{Motivation}

How to exploit the latent relationship between sensor signal dimensions is the main focus of the proposed approach. The signals belonging to different categories are supposed to have different inter-dimension dependent relationships which contain rich and discriminative information. This information is critical to improve the distinctive signal pattern discovery.

In practice, the sensor signal is often arranged as 1-D vector, which is less informative for the limited and fixed element arrangement. The elements order and the number of elements in each signal vector can affect the element dependency. In many real-world scenarios, the multimodal sensor data are associated with the practical placement. For example, the EEG data are concatenated following the distribution of biomedical EEG channels. Unfortunately, the practical sensor sequence, with the fixed order and number, may not be suitable for inter-dimension dependency analysis. Meanwhile, the optimal dimension sequence [Tan et al., 2015] varies with the sensor types and combinations. Therefore, we propose the following three techniques to amend these drawbacks.

First, we replicate and shuffle the input sensor signal vector on dimension-wise in order to provide as much latent dependency as possible among feature dimensions (Section 2.2).

Second, we introduce a focal zone as a selective attention mechanism, where the optimal inter-dimension dependency for each sample only depends on a small subset of features. Here, the focal zone is optimized by deep reinforcement learning which has been proved to be stable and wellperformed in policy learning (Section 2.3).

Third, we propose the WAS-LSTM classifier by extracting the distinctive inter-dimension dependency (Section 2.4).

\subsection{Data Replicate and Shuffle}

To provide more potential inter-dimension spatial dependencies, we propose a method called Replicate and Shuffle (RS). $\mathrm{RS}$ is a two-step feature transformation method which maps $\mathbf{x}_{i}$ to a higher dimensional space $\mathbf{x}_{i}^{\prime}$ with more complete ele- 
ment combinations:

$$
\mathbf{x}_{i} \in \mathbb{R}^{K} \rightarrow \mathbf{x}_{i}^{\prime} \in \mathbb{R}^{K^{\prime}}, K^{\prime}>K
$$

In the first step (Replicate), replicate $\mathbf{x}_{i}$ for $h=K^{\prime} \% K+1$ times where $\%$ denotes remainder operation. Then we get a new vector with length as $h * K$ which is not less than $K^{\prime}$; in the second step (Shuffle), we randomly shuffle the replicated vector in the first step and intercept the first $K^{\prime}$ element to generate $\mathbf{x}_{i}^{\prime}$. Theoretically, compared to $\mathbf{x}_{i}, \mathbf{x}_{i}^{\prime}$ contains more diverse and complete inter-dimension dependencies.

\subsection{Selective Attention Mechanism}

In the next process, we attempt to find the optimal dependency which includes the most distinctive information. But $K^{\prime}$, the length of $\mathbf{x}_{i}^{\prime}$, is too large and is computationally expensive. To balance the length and the information content, we introduce the attention mechanism [Cavanagh and others, 1992]. We introduce the attention mechanism to emphasize the informative fragment in $\mathbf{x}_{i}^{\prime}$ and denote the fragment by $\overline{\mathbf{x}}_{i}$, which is called focal zone. Suppose $\overline{\mathbf{x}}_{i} \in \mathbb{R}^{\bar{K}}$ and $\bar{K}$ denotes the length of the focal zone. For simplification, we continue denote the $k$-th element by $\overline{\mathbf{x}}_{i k}$ in the focal zone. To optimize the focal zone, we employ deep reinforcement learning as the optimization framework for its excellent performance in policy optimization [Mnih et al., 2015].

Overview. As shown in Figure 1, the focal zone optimization includes two key components: the environment (including state transition and reward model), and the agent. Three elements (the state $s$, the action $a$, and the reward $r$ ) are exchanged in the interaction between the environment and the agent. All of the three elements are customized based on the specific situation in this paper.

- The state $\mathcal{S}=\left\{s_{t}, t=0,1, \cdots, T\right\} \in \mathbb{R}^{2}$ describes the position of the focal zone, where $t$ denotes the time stamp. In the training, $s_{0}$ is initialized as $s_{0}=\left[\left(K^{\prime}-\right.\right.$ $\left.\bar{K}) / 2,\left(K^{\prime}+\bar{K}\right) / 2\right]$. Since the focal zone is a shifting fragment on 1-D $\mathbf{x}_{i}^{\prime}$, we design two parameters to define the state: $s_{t}=\left\{\right.$ start $_{i d x}^{t}$, end $\left._{i d x}^{t}\right\}$, where start $t_{i d x}^{t}$ and $e n d_{i d x}^{t}$ separately denote the start index and the end index of the focal zone ${ }^{1}$.

- The action $\mathcal{A}=\left\{a_{t}, t=0,1, \cdots, T\right\} \in \mathbb{R}^{4}$ describes which the agent could choose to act on the environment. In our case, we define 4 categories of actions for the focal zone (as described in the State Transition part in Figure 1): left shifting, right shifting, extend, and condense. Here at time stamp $t$, the state transition only choose one action to implement following the agent's policy $\pi: s_{t+1}=\pi\left(s_{t}, a_{t}\right)$.

- The reward $\mathcal{R}=\left\{r_{t}, t=0,1, \cdots, T\right\} \in \mathbb{R}$ is calculated by the reward model, which will be detailed later. The reward model $\Phi: r_{t}=\Phi\left(s_{t}\right)$ receives the current state and returns an evaluation as the reward.

- We employ the Dueling DQN (Deep Q Networks [Wang et al., 2015]) as the optimization policy $\pi\left(s_{t}, a_{t}\right)$,

${ }^{1}$ For example, for a random $\mathbf{x}_{i}^{\prime}=[3,5,8,9,2,1,6,0]$, the state $\left\{\right.$ start $\left._{i d x}^{t}=2, e n d_{i d x}^{t}=5\right\}$ is sufficient to determine the focal zone as $[8,9,2,1]$. which is enabled to learn the state-value function efficiently. Dueling DQN learns the Q value $V\left(s_{t}\right)$ and the advantage function $A\left(s_{t}, a_{t}\right)$ and combines them: $Q\left(s_{t}, a_{t}\right) \leftarrow V\left(s_{t}\right), A\left(s_{t}, a_{t}\right)$. The primary reason we employ a dueling DQN to optimize the focal zone is that it updates all the four $Q$ values $^{2}$ at every step while other policy only updates one $\mathrm{Q}$ value at each step.

Reward Model. Next, we detailedly introduce the design of the reward model for it is one crucial contribution of this paper. The purpose of reward model is to evaluate how the current state impact our final target which refers to the classification performance in our case. Intuitively, the state which can lead to the better classification performance should have a higher reward: $r_{t}=\mathcal{F}\left(s_{t}\right)$. As a result, in the standard reinforcement learning framework, the original reward model regards the classification accuracy as the reward. $\mathcal{F}$ refers to the WAS-LSTM. Note, WAS-LSTM focuses on the spatial dependency between different dimensions at the same timepoint while the normal LSTM focuses on the temporal dependency between a sequence of samples collected at different time-points. However, the WAS-LSTM requires considerable training time, which will dramatically increase the optimization time of the whole algorithm. In this section, we propose an alternative method to calculate the reward: construct a new reward function $r_{t}=\mathcal{G}\left(s_{t}\right)$ which is positively related with $r_{t}=\mathcal{F}\left(s_{t}\right)$. Therefore, we can employ $\mathcal{G}$ to replace $\mathcal{F}$. Then, the task is changed to construct a suitable $\mathcal{G}$ which can evaluate the inter-dimension dependency in the current state $s_{t}$ and feedback the corresponding reward $r_{t}$. We propose an alternative $\mathcal{G}$ composed by three components: the autoregressive model [Akaike, 1969] to exploit the inter-dimension dependency in $\mathbf{x}_{i}^{\prime}$, the Silhouette Score [Laurentini, 1994] to evaluate the similarity of the autoregressive coefficients, and the reward function based on the silhouette score.

The autoregressive model [Akaike, 1969] receives the focal zone $\overline{\mathbf{x}}_{i}$ and specifies that how the last variable depends on its own previous values.

$$
\bar{x}_{i \bar{K}}=\sum_{j=1}^{p} \varphi_{j} \bar{x}_{i(\bar{K}-j)}+C+\bar{\varepsilon}
$$

where $p$ is the order of the autoregressive model, $C$ indicates a constant, and $\bar{\varepsilon}$ indicates the withe noise. From this equation, we can infer that the autoregressive coefficient $\boldsymbol{\varphi}=\left\{\varphi_{j}, j=1,2, \cdots, p\right\} \in \mathbb{R}^{p}$ incorporates the dependent relationship in the focal zone. Then, to evaluate how rich information is taken in the $\varphi$, we employ silhouette score [Lovmar et al., 2005] to interpret the consistence of $\varphi$. The higher score $s s_{t}$ indicates the focal zone in state $s_{t}$ contains more inter-dimension dependency, which means $\overline{\mathbf{x}}_{i}$ is easier to be classified by the classifier in the next stage. At last, based on the $s s_{t} \in[-1,1]$, we design a reward function:

$$
r_{t}=\frac{e^{s s_{t}+1}}{e^{2}-1}-\beta \frac{\bar{K}}{K^{\prime}}
$$

The function contains two parts, the first part is a normalized exponential function with the exponent $s s_{t}+1 \in[0,1]$,

\footnotetext{
${ }^{2}$ Since we have four actions in $a_{t}$, the $Q\left(s_{t}, a_{t}\right)$ contains $4 \mathrm{Q}$ values. The arrangement is similar with the one-hot label.
} 
this part encourages the reinforcement learning algorithm to search the better $s_{t}$ which leads to a higher $s s_{t}$. The motivation of the exponential function is that: the reward growth rate is increasing with the silhouette score's increase ${ }^{3}$. The second part is a penalty factor for the focal zone length to keep the bar shorter and the $\beta$ is the penalty coefficient.

In summary, the aim of focal zone optimization is to learn the optimal focal zone $\overline{\mathbf{x}}_{i}^{*}$ which can lead to the maximum reward. The optimization totally iterates $N=n_{e} * n_{s}$ times where $n_{e}$ and $n_{s}$ separately denote the number of episodes and steps [Wang et al., 2015]. $\varepsilon$-greedy method [Tokic, 2010] is employed in the state transition.

\subsection{Weighted Average Spatial LSTM Classifier}

In this section, we propose Weighted Average Spatial LSTM classification for two purposes. The first attempt is to capture the cross-relationship among feature dimensions in the optimized focal zone $\overline{\mathbf{x}}_{i}^{*}$. The LSTM-based classifier is widely used for its excellent sequential information extraction ability which is approved in several research areas such as natural language processing [Gers and Schmidhuber, 2001; Sundermeyer et al., 2012]. Compared to other commonly employed spatial feature extraction methods, such as Convolutional Neural Networks, LSTM is less depends on the hyperparameters setting. However, the traditional LSTM focuses on the temporal dependency among a sequence of samples. Technically, the input data of traditional LSTM is 3-D tensor shaped as $\left[n_{b}, n_{t}, \bar{K}\right]$ where $n_{b}$ and $n_{s}$ denote the batch size and the number of temporal sample, separately. The WAS-LSTM aims to capture the dependency among various dimensions at one temporal point, therefore, we set $n_{t}=1$ and transpose the input data as: $\left[n_{b}, n_{t}, \bar{K}\right] \rightarrow\left[n_{b}, \bar{K}, n_{t}\right]$.

The second advantage of WAS-LSTM is that it could stabilize the performance of LSTM via moving average method [Lipton et al., 2015]. Specifically, we calculate the LSTM outputs $\mathbf{O}_{i}$ by averaging the past two outputs instead of only the final one (Figure 1):

$$
\mathbf{O}_{i}=\left(\mathbf{O}_{i(\bar{K}-1)}+\mathbf{O}_{i \bar{K}}\right) / 2
$$

The predicted label is calculated by $y_{i}^{\prime}=\mathcal{L}\left(\overline{\mathbf{x}}_{i}^{*}\right)$ where $\mathcal{L}$ denotes the LSTM algorithm. $\ell_{2}$-norm (with parameter $\lambda$ ) is adopted as regularization to prevent overfitting. The sigmoid activation function is used on hidden layers. The loss function is cross-entropy and is optimized by the AdamOptimizer algorithm [Kingma and $\mathrm{Ba}, 2014]$.

\section{Experiments}

In this section, we evaluate the proposed approach over 3 sensor signal datasets (separately collected by EEG headset, environmental sensor, and wearable sensor) including 2 widely used public datasets and 2 limited but more practical local datasets. Firstly we describe the details of each dataset. Secondly, we demonstrate the effectiveness and robustness by comparing the performance of our approach to baselines and state-of-the-art. Lastly, we provide the efficiency of the alternative reward model designed in Section 2.3.

${ }^{3}$ For example, for the same silhouette score increment $0.1, s s_{t}$ : $0.9 \rightarrow 1.0$ can earn higher reward increment than $s s_{t}: 0.1 \rightarrow 0.2$.

\begin{tabular}{llllllll}
\hline Datasets & Type & Task & \#-S & \#-C & Samples & \#-D & S-rate (Hz) \\
\hline EID & EEG & PID & 8 & 8 & 168,000 & 14 & 128 \\
RSSI & RFID & AR & 6 & 21 & 3,100 & 12 & 2 \\
PAMAP2 & IMU & AR & 9 & 8 & 120,000 & 14 & 100 \\
\hline
\end{tabular}

Table 1: Datasets description. PID, AR, and S-rate denote Person Identification, Activity Recognition, and Sampling rate, respectively. \#-S, \#-C, \#-D separately denote the number of subjects, classes, and dimensions.

\begin{tabular}{|c|c|c|c|c|c|}
\hline & \multirow{2}{*}{ Methods } & \multicolumn{4}{|c|}{ EID Dataset } \\
\hline & & Acc & Pre & Rec & F1 \\
\hline \multirow{5}{*}{$\begin{array}{c}\text { Non- } \\
\text { DL }\end{array}$} & SVM & 0.1438 & 0.1653 & 0.1545 & 0.1445 \\
\hline & $\mathbf{R F}$ & 0.9365 & 0.9261 & 0.9142 & 0.9457 \\
\hline & KNN & 0.9413 & 0.9471 & 0.9298 & 0.9511 \\
\hline & AB & 0.2518 & 0.2684 & 0.2491 & 0.2911 \\
\hline & LDA & 0.1485 & 0.1524 & 0.1358 & 0.1479 \\
\hline \multirow{5}{*}{ DL } & LSTM & 0.4315 & 0.5132 & 0.4278 & 0.4532 \\
\hline & GRU & 0.4314 & 0.455 & 0.4288 & 0.4218 \\
\hline & 1-D CNN & 0.8031 & 0.8127 & 0.805 & 0.8278 \\
\hline & WAS-LSTM & 0.9518 & 0.9657 & 0.9631 & 0.9658 \\
\hline & Ours & 0.9621 & 0.9618 & 0.9615 & 0.9615 \\
\hline
\end{tabular}

Table 2: Comparison of EID

\subsection{Datasets}

More details refer to Table 1.

- EID. The EID (EEG ID identification) is collected in a constrained setting where 8 subjects ( 5 males and 3 females) aged $26 \pm 2$. EEG signal monitors the electrical activity of the brain. This dataset gathers the raw EEG signals by Emotiv EPOC+ headset with 14 channels at the sampling rate of $128 \mathrm{~Hz}$.

- RSSI. The RSSI (Radio Signal Strength Indicator) [Yao et al., 2018] collects the signals from passive RFID tags. 21 activities, including 18 ADLs (Activity of Daily Living) and 3 abnormal falls, are performed by 6 subject aged $25 \pm 5$. RSSI measures the power present in a received radio signal, which is a convenient environmental measurement in ubiquitous computing.

- PAMAP2. The PAMAP2 [Fida et al., 2015] is collected by 9 participants ( 8 males and 1 females) aged $27 \pm 3$. 8 ADLs are selected as a subset of our paper. The activity is measured by 1 IMU attached to the participants' wrist. The IMU collects sensor signal with 14 dimensions including two 3 -axis accelerometers, one 3 -axis gyroscopes, one 3-axis magnetometers and one thermometer.

\subsection{Results}

In this section, we compare the proposed approach with baselines and the state-of-the-art methods. Our method focuses on the focal zone which is optimized by deep reinforcement learning and then explores the dependency between sensor signal elements by a deep learning classifier. All the three datasets are randomly split into the training set $(90 \%)$ and the testing set $(10 \%)$. Each sample is one sensor vector recording collected at one time point. Through the previous experimental tuning and the Orthogonal Array based hyper-parameters tuning method [Zhang et al., 2017], the hyper-parameters are set as following. In the selective attention learning: the order of autoregressive is $3 ; \bar{K}=128$, the Dueling DQN has 4 
Proceedings of the Twenty-Seventh International Joint Conference on Artificial Intelligence (IJCAI-18)

\begin{tabular}{|c|c|c|c|c|c|}
\hline & \multirow{2}{*}{ Methods } & \multicolumn{4}{|c|}{ RSSI Dataset } \\
\hline & & Acc & Pre & Rec & F1 \\
\hline \multirow{5}{*}{$\begin{array}{l}\text { Non- } \\
\text { DL }\end{array}$} & SVM & 0.8918 & 0.8924 & 0.8908 & 0.8805 \\
\hline & RF & 0.9614 & 0.9713 & 0.9652 & 0.9624 \\
\hline & KNN & 0.9612 & 0.9628 & 0.9618 & 0.9634 \\
\hline & AB & 0.4704 & 0.4125 & 0.4772 & 0.3708 \\
\hline & LDA & 0.8842 & 0.8908 & 0.8845 & 0.8802 \\
\hline \multirow{5}{*}{ DL } & LSTM & 0.7421 & 0.6505 & 0.6132 & 0.6858 \\
\hline & GRU & 0.7049 & 0.7728 & 0.6584 & 0.6915 \\
\hline & 1-D CNN & 0.9714 & 0.9676 & 0.9635 & 0.9645 \\
\hline & WAS-LSTM & 0.9553 & 0.9533 & 0.9545 & 0.9592 \\
\hline & Ours & 0.9838 & 0.9782 & 0.9669 & 0.9698 \\
\hline
\end{tabular}

Table 3: Comparison of RSSI

\begin{tabular}{|c|c|c|c|c|c|}
\hline & \multirow{2}{*}{ Methods } & \multicolumn{4}{|c|}{ PAMAP2 Dataset } \\
\hline & & Acc & Pre & $\operatorname{Rec}$ & F1 \\
\hline \multirow{5}{*}{$\begin{array}{c}\text { Non- } \\
\text { DL }\end{array}$} & SVM & 0.7492 & 0.7451 & 0.7522 & 0.7486 \\
\hline & $\mathbf{R F}$ & 0.9817 & 0.9893 & 0.9711 & 0.9801 \\
\hline & KNN & 0.9565 & 0.9651 & 0.9625 & 0.9638 \\
\hline & $\mathbf{A B}$ & 0.5776 & 0.4298 & 0.5814 & 0.4942 \\
\hline & LDA & 0.7127 & 0.7175 & 0.7298 & 0.7236 \\
\hline \multirow{3}{*}{ DL } & LSTM & 0.7925 & 0.7487 & 0.7478 & 0.7482 \\
\hline & GRU & 0.8625 & 0.8515 & 0.8349 & 0.8431 \\
\hline & 1-D CNN & 0.9819 & 0.9715 & 0.9721 & 0.9718 \\
\hline \multirow{6}{*}{$\begin{array}{l}\text { State- } \\
\text { of-the } \\
\text {-Arts }\end{array}$} & [Fida et al., 2015] & 0.96 & - & - & - \\
\hline & $\begin{array}{l}\text { [Chowdhury et al., } \\
2017]\end{array}$ & 0.8488 & - & - & 0.841 \\
\hline & [Erfani et al., 2017] & 0.967 & - & - & - \\
\hline & [Zheng et al., 2014] & 0.9336 & - & - & - \\
\hline & WAS-LSTM & 0.9821 & 0.9981 & 0.9459 & 0.9713 \\
\hline & Ours & 0.9882 & 0.9804 & 0.9756 & 0.9780 \\
\hline
\end{tabular}

Table 4: Comparison of PAMAP2

lays and the node number in each layer are: 2 (input layer), 32 (FCL), $4\left(A\left(s_{t}, a_{t}\right)\right)+1\left(V\left(s_{t}\right)\right), 4$ (output). The decay parameter $\gamma=0.8, n_{e}=n_{s}=50, N=2,500, \epsilon=0.2$, learning rate $=0.01$, memory size $=2000$, length penalty coefficient $\beta=0.1$, and the minimum length of focal zone is set as 10. In the deep learning classifier: the node number in the input layer equals to the number of feature dimensions, three hidden layers with 164 nodes, two layers of LSTM cells and one output layer. The learning rate $=0.001, \ell_{2}$-norm coefficient $\lambda=0.001$, forget bias $=0.3$, batch size $=9$, and iterate for 1000 iterations.

Tables $2 \sim 4$ show the classification metrics comparison between our approach and baselines including Non-DL and DL baselines. Since the EID and RSSI are local datasets, we only compare with state-of-the-art over the public dataset PAMAP2. Table 4 shows that our approach achieves the highest accuracy on both datasets. DL represents deep learning. The notation and hyper-parameters of the baselines are listed here. RF denotes Random Forest, AdaB denotes Adaptive Boosting, LDA denotes Linear Discriminant Analysis. In addition, the key parameters of the baselines are listed here: Linear SVM $(C=1), \operatorname{RF}(n=200)$, KNN $(k=3)$. In LSTM, $n_{\text {steps }}=5$, another set is the same as the WAS-LSTM classifier, along with the GRU (Gated Recurrent Unit [Chung et al., 2014]). The CNN works on sensor data and contains 2 stacked convolutional layers (both with stride $[1,1]$, patch $[2,2]$, zero-padding, and the depth are 4 and 8 , separately.) and followed by one pooling layer (stride $[1,2]$, zero-padding) and one fully connected layer (164 nodes). Relu activation function is employed in the CNN. The results from Tables $2 \sim 4$ show that:

- Our approach outperforms all the baselines and the stateof-the-arts over all the local and public datasets ranging from EEG, RFID to wearable IMU sensors;

- The sensor spatial based WAS-LSTM classifier achieves a high-level performance, which indicates the method that extracting inter-dimension dependency for classification is effective;

- Our method (WAS-LSTM with focal zone) performs better than WAS-LSTM, which illustrates that the learned informative attention is effective.

To have a closer observation, the CM (confusion matrix) and the ROC curves (including the AUC score) of the datasets are reported in Figure 2. The CMs illustrate that the robustness of the proposed approach keeps high accuracy even over few samples and numerous categories.

\subsection{Reward Model Efficiency Demonstration}

In this paper, we propose a new reward model to replace the original reward model: $\mathcal{G} \rightarrow \mathcal{F}$. The original $\mathcal{F}$, in our case, refers to the WAS-LSTM classifier (Section 2.4), intuitively. $\mathcal{F}$ requires a large amount of training time to find the optimal focal zone $\overline{\mathrm{x}}^{*}$. Take the EID dataset as an example, $\mathcal{F}$ needs around $4000 \mathrm{sec}$ on the Titan X (Pascal) GPU for each step while the whole focal zone optimization contains $N$ $(N>2000)$ iterations. Therefore, to save training time, we attempt to employ $\mathcal{G}$ to approximate $\mathcal{G}$ to update the reward. Thus, two prerequisites are demanded: 1) $\mathcal{G}$ should have high correlation with $\mathcal{F}$ to guarantee $\underset{\overline{\mathbf{x}}^{*}}{\arg \max } \mathcal{G} \approx \underset{\overline{\mathbf{x}}^{*}}{\arg \max } \mathcal{F} ; 2$ ) the training time of $\mathcal{G}$ should be shorter than $\mathcal{F}$. In this section, we demonstrate the two prerequisites by experimental analyzes.

First, on the focal zone optimization procedure on EID dataset, we conduct an experiment to measure a batch of data pairs of the reward (represents the reward of $\mathcal{G}$ ) and the WASLSTM classifier accuracy (represents the reward of $\mathcal{F}$ ). The relationship between the reward and the accuracy is shown in Figure 3. The figure illustrates that the accuracy has an approximately linear relationship with the reward. The correlations coefficient is 0.8258 (with p-value as 0.0115 ), which demonstrates the accuracy and reward are highly positive related. As a result, we can estimate $\arg \max \mathcal{F}$ by $\arg \max \mathcal{G}$. Moreover, another experiment is carried on to measure the single step training time of two reward models $\mathcal{G}$ and $\mathcal{F}$. The training times are marked as $\mathrm{T} 1$ and $\mathrm{T} 2$, respectively. Figure 4 qualitatively shows that $\mathrm{T} 2$ is much higher than T1 ( 8 states represent 8 different focal zones). Quantitatively, the sum of T1 over 8 states is $35237.41 \mathrm{sec}$ while the sum of $\mathrm{T} 2$ is $601.58 \mathrm{sec}$. This results demonstrate that the proposed approach, designing a $\mathcal{G}$ to approximate and estimate the $\mathcal{F}$, saves $\mathbf{9 8 . 3} \%$ training time in focal zone optimization.

\subsection{Discussions}

In this section, we discuss several characteristics of the proposed approach. 


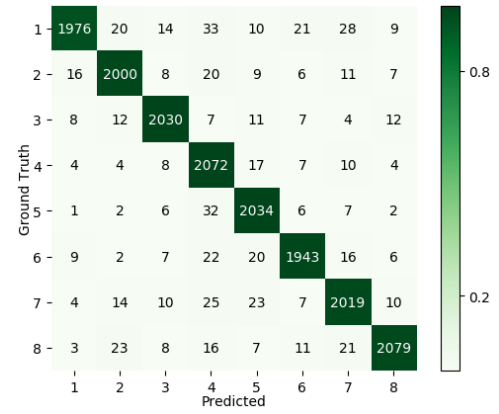

(a) EID CM

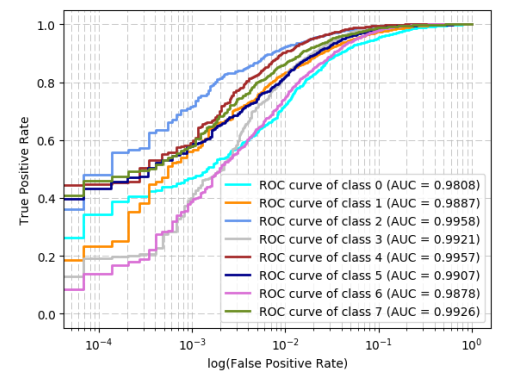

(d) EID ROC

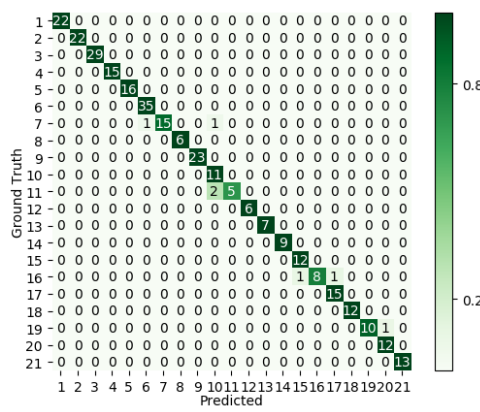

(b) RSSI CM

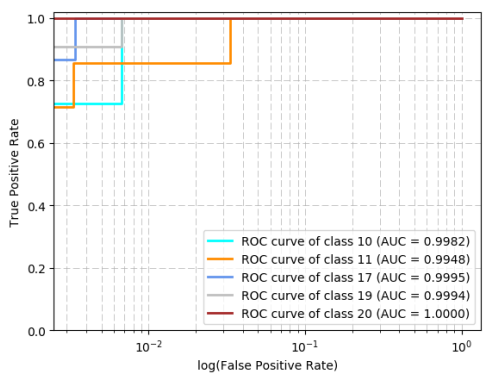

(e) RSSI ROC

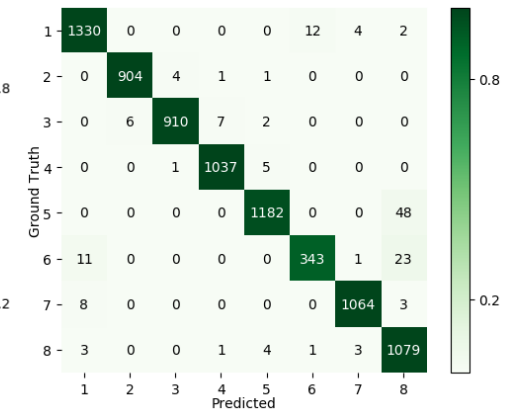

(c) PAMAP CM

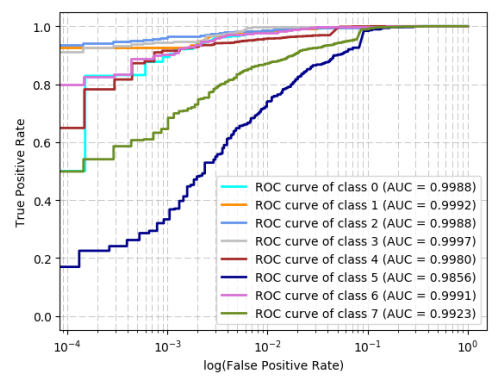

(f) PAMAP ROC

Figure 2: Confusion matrix and ROC curves of three datasets. CM denotes confusion matrix. The RSSI dataset overall contains 21 classes and we only select several representative classes.
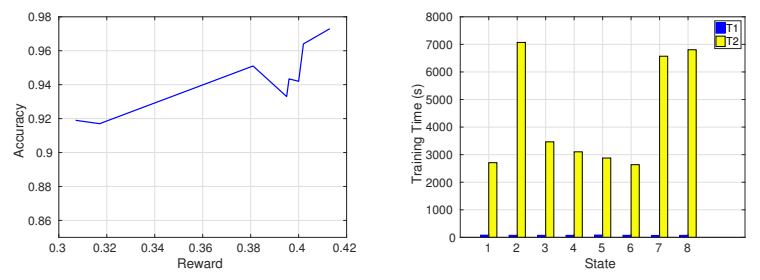

Figure 3: The relationship between the classifier accuracy and the reward. The correlationship coefficient is 0.8258 while the p-value is 0.0115 .
Figure 4: Reward model training time in various states. $\mathrm{T} 1$ and T2 separately denote the training time in reward model $\mathcal{G}$ and $\mathcal{F}$.

First, we propose a robust, universal, and adaptive classification framework which can efficiently deal with multimodality sensor data. Specifically, our approach works better on high-dimensional feature space in that the information of inter-dimension dependency is richer.

In addition, we propose a novel idea that adopts an alternative reward model to estimate and replace the original reward model. In this way, the disadvantages of the original model, such as expensive computation, can be eliminated. The key is to keep the reward produced by the new model highly related to the original reward. The higher correlation coefficient, the better. This sheds light on the possible combination of deep learning classifier and reinforcement learning.

Nevertheless, one weakness is that the reinforcement learning policy only works well in the specific environment in which the model is trained. The dimension indices should

be consistent in training and testing stages. Various policies should be trained according to different sensor combinations.

Furthermore, the proposed WAS-LSTM directly focuses on the dependency among the sensor dimensions and can produce a predicted label for each point. This provides the foundation for the quick-reaction online detection and other applications which require instantaneous detection. However, this reuires a enough number of signal dimensions to carry sufficient information for the aim of accurately recognition.

\section{Conclusion}

In this paper, we present a robust and efficient multi-modality sensor data classification framework which integrates selective attention mechanism, deep reinforcement learning, and WAS-LSTM classification. In order to boost the chance of inter-dimension dependency in sensor features, we replicate and shuffle the sensor data. Additionally, the optimal spatial dependency is required for high-quality classification, for which we introduce the focal zone with attention mechanism. Furthermore, we extended the LSTM to exploit the crossrelationship among spatial dimensions, which is called WASLSTM, for classification. The proposed approach is evaluated on three different sensor datasets, namely, EEG, RFID and wearable IMU sensors. The experimental results show that our approach outperforms the state-of-the-art baselines. Moreover, the designed reward model saves $\mathbf{9 8 . 3 \%}$ of the training time in reinforcement learning. 


\section{References}

[Akaike, 1969] Hirotugu Akaike. Fitting autoregressive models for prediction. Annals of the institute of Statistical Mathematics, 21(1):243-247, 1969.

[Bigdely-Shamlo et al., 2015] Nima Bigdely-Shamlo, Tim Mullen, Christian Kothe, Kyung-Min Su, and Kay A Robbins. The prep pipeline: standardized preprocessing for large-scale eeg analysis. Frontiers in neuroinformatics, 9:16, 2015.

[Cavanagh and others, 1992] Patrick Cavanagh et al. Attention-based motion perception. Science, 257(5076):1563-1565, 1992.

[Chowdhury et al., 2017] Alok Chowdhury, Dian Tjondronegoro, Vinod Chandran, and Stewart Trost. Physical activity recognition using posterior-adapted class-based fusion of multi-accelerometers data. IEEE Journal of Biomedical and Health Informatics, 2017.

[Chung et al., 2014] Junyoung Chung, Caglar Gulcehre, KyungHyun Cho, and Yoshua Bengio. Empirical evaluation of gated recurrent neural networks on sequence modeling. arXiv preprint arXiv:1412.3555, 2014.

[Erfani et al., 2017] Sarah M Erfani, Mahsa Baktashmotlagh, Masud Moshtaghi, Vinh Nguyen, Christopher Leckie, James Bailey, and Kotagiri Ramamohanarao. From shared subspaces to shared landmarks: A robust multi-source classification approach. In $A A A I$, pages 1854-1860, 2017.

[Fida et al., 2015] Benish Fida, Daniele Bibbo, Ivan Bernabucci, Antonino Proto, Silvia Conforto, and Maurizio Schmid. Real time event-based segmentation to classify locomotion activities through a single inertial sensor. In MOBIHEALTH, pages 104-107, 2015.

[Gers and Schmidhuber, 2001] Felix A Gers and E Schmidhuber. Lstm recurrent networks learn simple context-free and context-sensitive languages. IEEE Transactions on Neural Networks, 12(6):1333-1340, 2001.

[Gers et al., 1999] Felix A Gers, Jürgen Schmidhuber, and Fred Cummins. Learning to forget: Continual prediction with lstm. 1999.

[Kamburugamuve et al., 2015] Supun Kamburugamuve, Leif Christiansen, and Geoffrey Fox. A framework for real time processing of sensor data in the cloud. Journal of Sensors, 2015, 2015.

[Kingma and Ba, 2014] Diederik Kingma and Jimmy Ba. Adam: A method for stochastic optimization. arXiv preprint arXiv:1412.6980, 2014.

[Laurentini, 1994] Aldo Laurentini. The visual hull concept for silhouette-based image understanding. IEEE Transactions on pattern analysis and machine intelligence, 16(2):150-162, 1994.

[Lipton et al., 2015] Zachary C Lipton, David C Kale, Charles Elkan, and Randall Wetzel. Learning to diagnose with lstm recurrent neural networks. arXiv preprint arXiv:1511.03677, 2015.
[Lovmar et al., 2005] Lovisa Lovmar, Annika Ahlford, Mats Jonsson, and Ann-Christine Syvänen. Silhouette scores for assessment of snp genotype clusters. BMC genomics, 6(1):35, 2005.

[Markham and Townshend, 1981] BL Markham and JRG Townshend. Land cover classification accuracy as a function of sensor spatial resolution. 1981.

[Mnih et al., 2015] Volodymyr Mnih, Koray Kavukcuoglu, David Silver, Andrei A Rusu, Joel Veness, Marc G Bellemare, Alex Graves, Martin Riedmiller, Andreas K Fidjeland, Georg Ostrovski, et al. Human-level control through deep reinforcement learning. Nature, 518(7540):529, 2015.

[Sundermeyer et al., 2012] Martin Sundermeyer, Ralf Schlüter, and Hermann Ney. Lstm neural networks for language modeling. In Thirteenth Annual Conference of the International Speech Communication Association, 2012.

[Tan et al., 2015] Ming Tan, Cicero dos Santos, Bing Xiang, and Bowen Zhou. Lstm-based deep learning models for non-factoid answer selection. arXiv preprint arXiv:1511.04108, 2015.

[Tokic, 2010] Michel Tokic. Adaptive $\varepsilon$-greedy exploration in reinforcement learning based on value differences. In Annual Conference on Artificial Intelligence, pages 203 210. Springer, 2010.

[Wang et al., 2015] Ziyu Wang, Tom Schaul, Matteo Hessel, Hado Van Hasselt, Marc Lanctot, and Nando De Freitas. Dueling network architectures for deep reinforcement learning. arXiv preprint arXiv:1511.06581, 2015.

[Yao et al., 2018] Lina Yao, Quan Z Sheng, Xue Li, Tao Gu, Mingkui Tan, Xianzhi Wang, Sen Wang, and Wenjie Ruan. Compressive representation for device-free activity recognition with passive rfid signal strength. IEEE Transactions on Mobile Computing, 17(2):293-306, 2018.

[Zhang et al.,] Xiang Zhang, Lina Yao, Quan Z Sheng, Salil S Kanhere, Tao Gu, and Dalin Zhang. Converting your thoughts to texts: Enabling brain typing via deep feature learning of eeg signals. In PerCom 2018.

[Zhang et al., 2017] Xiang Zhang, Lina Yao, Chaoran Huang, Quan Z Sheng, and Xianzhi Wang. Intent recognition in smart living through deep recurrent neural networks. In International Conference on Neural Information Processing, pages 748-758. Springer, 2017.

[Zhang et al., 2018] Dalin Zhang, Lina Yao, Xiang Zhang, Sen Wang, Weitong Chen, and Robert Boots. Eeg-based intention recognition from spatio-temporal representations via cascade and parallel convolutional recurrent neural networks. In AAAI, 2018.

[Zheng et al., 2014] Yi Zheng, Qi Liu, Enhong Chen, Yong $\mathrm{Ge}$, and $\mathrm{J}$ Leon Zhao. Time series classification using multi-channels deep convolutional neural networks. In International Conference on Web-Age Information Management, pages 298-310. Springer, 2014. 